\title{
Van Hove Singularities in disordered multichannel quantum wires and nanotubes
}

\author{
S. Hügle and R. Egger \\ Institut für Theoretische Physik, Heinrich-Heine-Universität, D-40225 Düsseldorf, Germany
}

(Date: November 8, 2018)

\begin{abstract}
We present a theory for the van Hove singularity (VHS) in the tunneling density of states (TDOS) of disordered multichannel quantum wires, in particular multi-wall carbon nanotubes. We assume close-by gates which screen off electronelectron interactions. Diagrammatic perturbation theory within the non-crossing approximation yields analytical expressions governing the disorder-induced broadening and shift of VHS's as new subbands are opened. This problem is nontrivial because the (lowest-order) Born approximation breaks down close to the VHS. Interestingly, compared to the bulk case, the boundary TDOS shows drastically altered VHS, even in the clean limit.
\end{abstract}

Van Hove singularities (VHS's) in the thermodynamif density of states (DOS) have been predicted in 1953日 and were observed in many experiments since then. The DOS for a $d$-dimensional system with dispersion relation $E(\vec{k})$ can be written as an integral over the Fermi surface, $\nu(E)=(2 \pi)^{-d} \int d S /\left|\nabla_{\vec{k}} E(\vec{k})\right|$. The quantity in the denominator is basically the group velocity. Due to symmetries in a crystal, the group velocity may vanish at certain momenta, resulting in a divergent integrand. This divergence is integrable in three dimensions, and typically leads to a finite DOS. In lower dimensions, however, very pronounced VHS appear. In this paper, we focus on the one-dimensional (1D) limit, where the VHS diverges like $\nu(E) \sim 1 / \sqrt{E-E_{n}}$ in a clean system when the energy $E$ approaches the threshold $E_{n}$ from above. Therefore VHS's appear as sharp features in the energy-dependent DOS reflecting the onset of new active subbands. Similar VHS's exist for the tunneling density of states (TDOS) measured at some location $x$ along the system,

$$
\nu(E, x)=\frac{\operatorname{Re}}{\pi \hbar} \int_{0}^{\infty} d t e^{i E t / \hbar}\left\langle\psi(x, t) \psi^{\dagger}(x, 0)\right\rangle,
$$

where $\psi(x, t)$ is the electron operator. The TDOS is easily accessible experimentally by measuring the conductance through a weak link or a tunnel junction. Typically, it is obtained by means of scanning tunneling spectroscopy (STS). 2.3 In general, one has to carefully distinguish between the DOS and the TDOS. While the DOS is a thermodynamic property of the system, the TDOS is a local property and therefore depends on the position. In the "bulk" limit, the DOS and the (possibly coarse-grained) TDOS are expected to be identical, but near a boundary they can strongly differ. In the present paper we address the question: How is the VHS modified by disorder? This question is of importance for the interpretation of experiments on multi-wall nanotubes (MWNTs). A MWNT consists of a few concentrically arranged graphite shells, with a typical outermostshell radius $R \approx 5$ to $20 \mathrm{~nm}$ and lengths of up to $1 \mathrm{~mm}$ As demonstrated by magnetoresistance measurements, electronic transport occurs only through the outermost shell and exhibits the typical fingerprints of diffusive behavior while single-wall nanotubes (SWNTs) are ballistic 1D quantum wires 5 The observation of VHS's in recent STS experiments on SWNTs on a metallic substrate represents a direct proof for a $1 \mathrm{D}$ band structure.6.0. $\mathrm{B}$ In particular, the predicted $1 / \sqrt{E-E_{n}}$ behavior of ballistic 1D wires has been observed.

Since in MWNTs the incommensurate inner-shell ionic potential acts on electrons in the outermost shell, the resulting quasi-periodic potential effectively acts as a random potential. 9 We then want to understand how VHS's develop with increasing amounts of disorder. By intentionally damaging MWNTs, e.g. by fast ion bombarding, any level of disorder may be realized experimentally to test our predictions below. For typical MWNTs with mean free path $l \approx 2 \pi R$, the characteristic subband features of the TDOS should still be present, albeit considerably broadened and possibly shifted. This question is clearly also of relevance to other quasi-1D quantum wires such as long chain molecules. Previous experiments on intrinsically (hole-) doped MWNTs reported by Bachtold et al. 10 have observed power-law zero bias anomalies due to electron-electron interactions. 9 Here we focus on a completely different scenario, where interactions are screened off by working on a metallic substrate which is typical for STS measurements. Hence we treat only the non-interacting problem. Then spin only contributes trivial factors of two and can be ignored. In addition, disorder-induced scattering between the two distinct gapless Fermi (K) points of the first Brillouin zone of the honeycomb lattice is expected to be largely suppressed, and we thus consider only one Fermi point. Since in typical STS experiments only the TDOS of the outer-most shell is probed, in the following an effective single-shell model is assumed, where inner shells only give rise to a disorder potential for outer-shell electrons.

Nanotubes (NTs) can be thought of as graphite sheets wrapped onto a cylinder. The low-energy theory of a clean graphite sheet is given by the 2D Dirac Hamiltonian. This graphite sheet is rolled up into a tube by enforcing periodic boundary conditions around the $y$ direction, the tube pointing along the $x$ direction. For later convenience, we define $\beta^{-1}=\hbar v_{F} / 2 \pi R$ with $v_{F}=8 \times 10^{5}$ 
$\mathrm{m} / \mathrm{s}$. (To simplify notation, we often set $\hbar=v_{F}=1$.) In the absence of disorder, we have

$$
H_{0}=\frac{1}{\beta} \sum_{n} \int \frac{d k}{2 \pi} \psi^{\dagger}(\vec{k})\left(\vec{\sigma} \cdot \vec{k}+M \sigma_{y}\right) \psi(\vec{k})
$$

with $\vec{k}=\left(k, \omega_{n}\right)$. The "Matsubara frequencies" $\omega_{n}$ given by $E_{n}=2 \pi n / \beta=\hbar \omega_{n}$ with integer $n$ arise due to the finite radius. $\psi$ is a two-component spinor, where the two components reflect the sublattice degree of freedom, as the honeycomb lattice has a basis containing two atoms, and the Pauli matrices $\vec{\sigma}=\left(\sigma_{x}, \sigma_{y}\right)$ act in this space. The "mass" $M$ is generally nonzero for NTs due to chirality effectsor an applied magnetic field $B$ parallel to the tube axis. 11 For a flux $\Phi$ (in units of the flux quantum), we get $M=2 \pi \Phi / \beta$. Since chirality effects can always be absorbed by an adjustment of $B$, we take $\Phi=B / B_{0}$ with $B_{0}=h /\left(e \pi R^{2}\right)$. The mass term in Eq. (11) couples in the same way as $k_{y}=\omega_{n}$, and hence we can replace

$$
\omega_{n} \rightarrow \omega_{n}+2 \pi \Phi / \beta=\left(n+B / B_{0}\right) / R
$$

in Eq. (1) to include the mass term. For integer $\Phi$, the system is obviously not influenced by the magnetic field, as we can simply shift $n$ to absorb $\Phi$. Next we discuss our modelling of disorder. On the one hand, there may be disorder-induced hopping events between nearest-neighbor sites on the honeycomb lattice via real or virtual states provided by the inner-shell ionic potential. Since hopping connects different sublattices, the resulting modulation of the hopping matrix element leads to a random gauge field. Gauge field disorder also captures the effects of topological defects in the outermost shell.12 However, in particular once the MWNT is intrinsically damaged, the dominant disorder mechanism should be due to direct impurity potential scattering processes which are diagonal in sublattice space,

$$
H_{V}=\int d \vec{x} V(\vec{x}) \psi^{\dagger}(\vec{x}) \psi(\vec{x}) .
$$

In what follows, we retain only disorder of the type (3). The scattering potential is taken as a static Gaussian random field with zero mean and $\left\langle V(\vec{x}) V\left(\vec{x}^{\prime}\right)\right\rangle=$ $\Delta_{V} \delta\left(\vec{x}-\vec{x}^{\prime}\right)$, where $\Delta_{V}=\hbar^{2} v_{F}^{2} \Delta / R$ defines the dimensionless disorder strength $\Delta$.

In addition to the bulk case, we also address the boundary TDOS arising when one tunnels into the end of a MWNT. In reality, the end TDOS could of course be quite complex due to the formation of bound states. A few lattice spacings away from the end, however, we expect that the situation can be described by a continuum model. Surprisingly, the presence of a boundary implies a drastic change in the TDOS even in the absence of interactions, namely a strong suppression of the TDOS close to the boundary. Since this can be demonstrated already in the absence of disorder, let us briefly discuss the clean limit. The TDOS at position $x$ and energy $E>0$ is $\nu(E, x)=-\operatorname{Im}_{\operatorname{Tr}_{\vec{k}, \sigma}} G(E, \vec{k}, x) / \pi$, where we have to trace over the "spin" and the momentum. We consider a semi-infinite $(x \geq 0)$ tube, assuming a hard-wall potential at the boundary $x=0$. The Greens function is then $G_{0}(E, \vec{k}, x)=2 \sin ^{2}(k x) /\left(E-\vec{\sigma} \cdot \vec{k}+i 0^{+}\right)$, and hence

$$
\begin{aligned}
\frac{\nu(E, x)}{\nu_{1 \mathrm{D}}} & =2|E| \\
& \times \sum_{n=-\infty}^{\infty} \frac{\sin ^{2}\left(x \sqrt{E^{2}-E_{n}^{2}}\right)}{\sqrt{E^{2}-E_{n}^{2}}} \Theta\left(E^{2}-E_{n}^{2}\right),
\end{aligned}
$$

where $\Theta$ is the Heaviside step function and $\nu_{1 \mathrm{D}}=1 / \pi \hbar v_{F}$ serves as natural unit for the TDOS (without spin and $\mathrm{K}$ point degeneracy). As one can see from Eq. (4) with (2), a gap is generally present. This gap varies as a function of $B$, and vanishes periodically with period $B_{0}$. Far away from the boundary, $\sin ^{2}\left(x \sqrt{E^{2}-E_{\eta}^{2}}\right) \rightarrow 1 / 2$, and we obtain the bulk TDOS (see also Ref. 13)

$$
\frac{\nu_{0}(E)}{\nu_{1 \mathrm{D}}}=|E| \sum_{n} \frac{\Theta\left(E^{2}-E_{n}^{2}\right)}{\sqrt{E^{2}-E_{n}^{2}}},
$$

which equals the thermodynamic DOS. The well-known $1 / \sqrt{E-E_{n}}$ VHS's in 1D appear at the onset of new subbands. Since the summation also includes negative values of $n$, a magnetic field in general causes a doubling of the VHS's, see Eq. (2). Sufficiently close to the boundary, however, we obtain a completely different result. From Eq. (4) we find the boundary TDOS

$$
\frac{\nu_{\text {end }}(E, x)}{\nu_{1 \mathrm{D}}}=2 x^{2}|E| \sum_{n} \Theta\left(E^{2}-E_{n}^{2}\right) \sqrt{E^{2}-E_{n}^{2}} .
$$

For $E \rightarrow 0$, this predicts $\nu(E) \sim E^{2}$ for $B=0$, in contrast to the finite boundary TDOS for a doped tube. This behavior can be traced back to the linear dispersion relation of Dirac fermions. More interestingly, the typical 1D VHS of the bulk TDOS is drastically altered close to the boundary. Instead of a divergence, the only sign of the opening of new subbands is a nonanalyticity at the threshold energy $E_{n}$, with a square-root energy dependence of the boundary TDOS above $E_{n}$. This possibly explaines the observation of Ref. 8 , where no divergencies were found in the tunneling spectrum at the end of a SWNT. We note that this phenomenon is quite general. For both Dirac and Schrödinger fermions, the exponent in the TDOS energy dependence close to a VHS changes by one when going from the bulk to the boundary limit. For $E \rightarrow 0$, however, for Dirac fermions, the exponent changes by two due to the special role of the energy $E=0$. The relevant crossover scale between bulk and boundary behavior of the TDOS depends on energy. Focussing on $E$ close to but above a given threshold energy $E_{n}$, this scale is $x^{*} \approx \hbar v_{F} / \sqrt{E^{2}-E_{n}^{2}}$. At zero temperature, the bulk limit is reached for $x \gg x^{*}$, and the boundary limit for $x \ll x^{*}$. For finite temperatures $T$, if the thermal scale $x_{T}=\hbar v_{F} / k_{B} T$ is smaller than $x^{*}$, one should replace $x^{*}$ by $x_{T}$. 
We study disordered MWNTs using diagrammatic perturbation theory within the non-crossing approximation (NCA). 44 The use of NCA for this problem requires some care. On the one hand, for 2D random Dirac fermions, diagrams with crossing impurity lines cause the same (logarithmic) singularities as rainbow diagrams in arbitrary orders of perturbation theory, 15 and crossing diagrams must be treated on the same footing as the rainbow ones. On the other hand, in the single-channel 1D limit, it is also well-known from exact calculations that crossed diagrams are in general as important as rainbow diagrams. Since MWNTs are in between the 1D and the 2D limit, studying the importance of crossed diagrams is therefore mandatory. The situation for MWNTs is fortunately quite different from both limits mentioned above. First, there are no logarithmic singularities appearing in the self energy expansion as in the 2D limit. Second, evalua tion of the simplest diagrams using standard methods 14 shows that rainbow diagrams are larger than crossed diagrams by a factor $4 \pi \nu(E) / \nu_{1 D}$. For the energy range of interest here, we are therefore entitled to compute the TDOS within NCA. We focus on the bulk case here and briefly comment on the boundary result later.

With $G_{0}(E, \vec{k})=\left(E-\vec{\sigma} \cdot \vec{k}+i 0^{+}\right)^{-1}$, the self energy to lowest order in $\Delta_{V}$ (Born approximation) takes the form $\Sigma^{(1)}(E)=-i \pi \Delta_{V} \nu_{0}(E)$, with $\nu_{0}(E)$ given by Eq. (5). Remarkably, the Born approximation breaks down in the vicinity of a VHS. Specifically, the second-order self energy contribution obeys

$$
\left|\frac{\Sigma^{(2)}(E)}{\Sigma^{(1)}(E)}\right|=\pi \Delta_{V} \partial_{E} \nu_{0}(E),
$$

which diverges for $E$ approaching $E_{n}$ from above. We therefore must address the higher-order contributions to the self energy. Even within NCA, since the perturbation expansion is asymptotic, we have to arrange the order of summation in a physically meaningful way to avoid familiar but unphysical divergencies and inconsistencies. This would also be important for analyzing diagrams beyond NCA, see Ref. 14. To achieve that, we follow the self-consistent iterative approach proposed by Lee 10 Within this approach, the self energy $\Sigma_{N}$ including all contributions up to $N$ th order $(N \geq 1)$ is $\Sigma_{N}(E)=\Delta_{V} \operatorname{Tr}_{\vec{k}, \sigma} G_{N-1}(E, \vec{k})$, with the corresponding Dyson equation $G_{N}^{-1}(E, \vec{k})=G_{0}^{-1}(E, \vec{k})-\Sigma_{N}(E)$. This form is then used to calculate the self energy $\Sigma_{N+1}$. In the limit $N \rightarrow \infty$, this procedure converges leading to

$$
\Sigma(E)=\Delta_{V} \operatorname{Tr}_{\vec{k}, \sigma} \frac{1}{G_{0}^{-1}(E, \vec{k})-\Sigma(E)},
$$

which has to be solved self-consistently for $\Sigma(E)$. One can check explicitly in each order that Eq. (]) reproduces all non-crossing diagrams correctly. The result can then be used to compute the TDOS, $\nu(E)=-\operatorname{Im} \Sigma(\mathrm{E}) / \pi \Delta_{\mathrm{V}}$. It is not possible to simply assume an energy-independent mean free path for all energies. The energy-dependent mean free time is $-\operatorname{Im} \Sigma(E)=\hbar / 2 \tau(E)$, and therefore also an energy-dependent mean free path $l(E)=v_{F} \tau(E)$ results.

From Eq. (7), we obtain $\left(\Sigma_{R}=\operatorname{Re} \Sigma\right)$

$$
\Sigma(E)=-i \Delta \frac{\hbar v_{F}}{R} \sum_{n} \frac{(E-\Sigma(E)) \operatorname{sgn}\left(E-\Sigma_{R}\right)}{\sqrt{(E-\Sigma(E))^{2}-E_{n}^{2}}},
$$

where correct units were restored for clarity. Equation (8) can easily be solved numerically. Once we know the self energy, the TDOS follows as

$$
\frac{\nu(E)}{\nu_{1 \mathrm{D}}}=\operatorname{Re} \sum_{n} \frac{(E-\Sigma(E)) \operatorname{sgn}\left(E-\Sigma_{R}\right)}{\sqrt{(E-\Sigma(E))^{2}-E_{n}^{2}}} .
$$

For $\Delta>0$, the finite imaginary part of $\Sigma$ in the denominator of (9) causes a broadening of the VHS, whereas the real part causes an energy shift of the peaks. For the numerical evaluation of Eq. (8), a cutoff for the band index $n$ has to be used, which is naturally given from the bandstructure. For instance, for armchair NTs, the number of subbands is given by $2 N=8 \pi R / \sqrt{3} a .17$ For $R=10 \mathrm{~nm}$, we get $N \approx 295$. The results are, however, not very sensitive to the precise choice of this cutoff. Equations (8) and (9) can be used to fit experimental data for the TDOS of MWNTs. Assuming that $R$ is known, since $\Delta$ is the only fit parameter, the disorder strength can then be determined directly from the TDOS which should allow for detailed comparison to STS experiments on MWNTs. This would provide precious information on the disorder strength.

Figure 1 shows the strong broadening of the VHS due to disorder. For sufficiently strong disorder, namely when motion around the circumference becomes diffusive, the peaks can even disappear completely above a certain energy which decreases with increasing $\Delta$. In the region where no VHS's are present, the TDOS apparently behaves like a power law, $\nu(E) \sim E^{\alpha}$, with $\alpha(\Delta) \leq 1$ This power law scaling holds remarkably well for $\Delta \leq 0.05$. Note that energies are not measured relative to $E_{F}$, and therefore this power law is unrelated to the findings of Ref. 10. Also it has a different origin than the power law found in the $2 \mathrm{D}$ aase that rests upon the inclusion of crossed diagrams. 0 In addition, the positions of the VHS's are shifted to smaller energies with increasing $\Delta$. This shift grows approximately linearly with $\Delta$, and the relative shift, compared to the position of the VHS in the clean system, can be up to $20 \%$ depending on the disorder strength. Since the radius of a NT is often determined from the relative positions of the VHS, this observation suggests that such interpretations need to be taken with some caution. The disorder-induced shift has to be taken into account to obtain correct results. With decreasing radius and for increasing order $n$ of the VHS, the relative shift becomes systematically smaller. Next, if we consider a fixed $\Delta$ and vary a magnetic field $B$ applied parallel to the tube axis, we find the situation depicted in Fig. 2. Again, there is a doubling of the VHS's, and the 
shift of the positions varies periodically with $B$. As in the clean case, there is a gap even in the presence of disorder. But due to the $\Delta$-dependent shift of the VHS towards smaller energies, the gap gets partially filled with states and is therefore smaller than in the clean case. However, magnetic field effects are only weakly affected by disorder. Finally, we briefly turn to the boundary limit. Since it is easy to generalize Eqs. (8) and (9) to obtain the boundary TDOS, we only mention the modifications compared to the clean case. The disordered boundary TDOS is suppressed compared to the clean case, where the suppression increases with increasing $\Delta$. The positions of the van Hove nonanalyticities at the opening of new subbands are shifted to higher energies, although the shift in the bulk case was to smaller energies. The form of the nonanalyticity is not significantly changed by disorder, but approximately retains the square-root energy dependence.

To conclude, we have calculated the TDOS of disordered multichannel quantum wires, with special emphasis on MWNTs. In the present theory, electron-electron interactions are supposed to be screened off by a metallic substrate or a close-by gate. Focussing on static disorder, within NCA a self-consistent non-pertubative summation of all diagrams for the self energy yields an analytical result for the disorder-broadened VHS's. For given radius of the MWNT, this result involves only one parameter (the disorder strength $\Delta$ ), which should allow for a detailed comparison to STS experiments on MWNTs, where the disorder strength can be tuned at will.

We thank A. O. Gogolin for useful discussions. Financial support by the DFG under the Gerhard-Hess program is acknowledged.

${ }^{1}$ L. van Hove, Phys. Rev. 89, 1189 (1953).

${ }^{2}$ J. Tersoff and D. R. Hamann, Phys. Rev. Lett. 50, 1998 (1983); Phys. Rev. B 31, 805 (1985).

${ }^{3}$ S. Hügle, R. Egger, and H. Grabert, Solid State Commun. 117, 93 (2001).

${ }^{4}$ A. Bachtold et al., Nature (London) 397, 673 (1999).

${ }^{5}$ S. J. Tans et al., Nature (London) 394, 761 (1997).

${ }^{6}$ J. W. G. Wildöer et al., Nature (London) 391, 59 (1998); T. W. Odom et al., ibid. 391, 62 (1998).

${ }^{7}$ L.C. Venema et al., Phys. Rev. B 62, 5238 (2000).

${ }^{8}$ P. Kim et al., Phys. Rev. Lett. 82, 1225 (1999).

${ }^{9}$ R. Egger and A. O. Gogolin, Phys. Rev. Lett. 87, 066401 (2001).

10 A. Bachtold et al., Phys. Rev. Lett. 87, 166801 (2001).

${ }^{11}$ C. L. Kane and E. J. Mele, Phys. Rev. Lett. 78, 1932 (1997).

12 J. González, F. Guinea, and M. A. H. Vozmediano, Phys. Rev. Lett. 69, 172 (1992); Phys. Rev. B 63, 134421 (2001).

${ }^{13}$ J. W. Mintmire and C. T. White, Phys. Rev. Lett. 81, 2506
(1998)

14 A. A. Abrikosov, L. P. Gorkov, and I. Dzyaloshinskii, Quantum Field Theoretical Methods in Statistical Physics (Pergamon, New York, 1965).

15 A. A. Nersesyan, A. M. Tsvelik, and F. Wenger, Phys. Rev. Lett. 72, 2628 (1994); Nucl. Phys. B 438, 561 (1995).

${ }^{16}$ P. A. Lee, Phys. Rev. Lett. 71, 1887 (1993).

${ }^{17}$ R.A. Jishi et al., J. Phys. Soc. Jpn. 63, 2252 (1994).

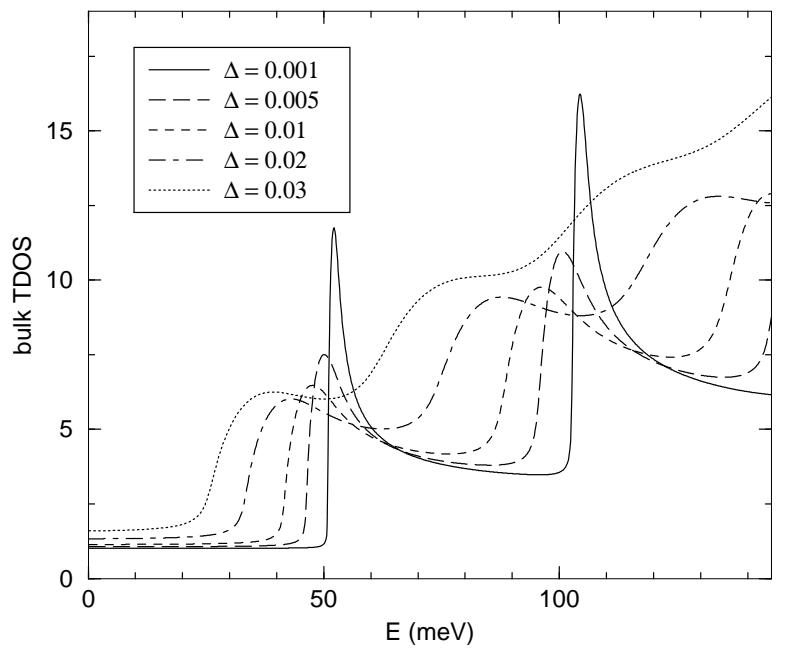

FIG. 1. Bulk TDOS of a disordered tube with $R=10 \mathrm{~nm}$ at $B=0$ for different values of $\Delta$. The broadening of the VHS with increasing $\Delta$ is clearly visible, as well as their shift towards smaller energies.

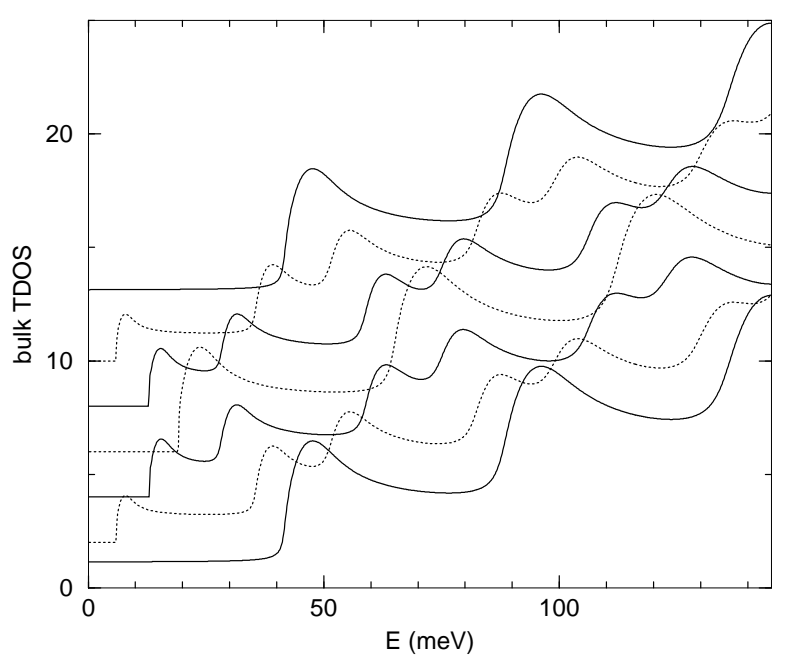

FIG. 2. Bulk TDOS for $\Delta=0.01, R=10 \mathrm{~nm}$ and different values of $B$. The curves corresponding to different $B$ are shifted vertically by the same amount for better visibility. The lowest curve corresponds to $B=0$, then $B$ increases in steps of $\Delta B=2.2 \mathrm{~T}$. Notice the opening and closing of a gap with period $B_{0}=13.2 \mathrm{~T}$. 